\title{
Médiévales
}

Langues, Textes, Histoire

61 | automne 2011

La chair des émotions

\section{Entre lapsus corporis et performance : fonctions des gestes somatiques dans l'expression des émotions dans la littérature altimédiévale}

Between lapsus corporis and performance: the functions of somatic gestures in the expression of emotions in the early Middle Ages literature

\section{Nira Pancer}

\section{OpenEdition}

Journals

Édition électronique

URL : https://journals.openedition.org/medievales/6253

DOI : 10.4000/medievales. 6253

ISSN : 1777-5892

\section{Éditeur}

Presses universitaires de Vincennes

\section{Édition imprimée}

Date de publication : 20 décembre 2011

Pagination : $39-53$

ISBN : 978-2-84292-337-2

ISSN : 0751-2708

\section{Référence électronique}

Nira Pancer, «Entre lapsus corporis et performance : fonctions des gestes somatiques dans l'expression des émotions dans la littérature altimédiévale », Médiévales [En ligne], 61 | automne 2011, mis en ligne le 17 janvier 2012, consulté le 23 avril 2022. URL : http://journals.openedition.org/ medievales/6253 ; DOI : https://doi.org/10.4000/medievales.6253 
Médiévales 61, automne 2011, p. 39-54

\author{
Nira PANCER
}

\title{
ENTRE LAPSUS CORPORIS ET PERFORMANCE: FONCTIONS DES GESTES SOMATIQUES DANS L'EXPRESSION DES ÉMOTIONS DANS LA LITTÉRATURE ALTIMÉDIÉVALE
}

De saint Augustin à saint Thomas d'Aquin, les philosophes du Moyen Âge ont toujours fait la part belle à l'expression corporelle et physiologique de l'émotion ${ }^{1}$. Cependant, de manière paradoxale, cette approche physique du phénomène émotionnel n'est pas franchement prégnante dans l'ensemble de la littérature médiévale. Larmes, sourires, rougissements, tremblements de rage ou de peur: les gestes somatiques qui viennent signifier l'état émotionnel des héros que célèbrent les auteurs épiques ne ponctuent qu'occasionnellement les récits des chansons de geste ${ }^{2}$, et cette tendance se fait plus marquée au fur et à mesure que l'on remonte dans le temps. Force est de constater que les littérateurs des premiers siècles médiévaux ( $\mathrm{VI}^{\mathrm{e}}-\mathrm{VIII}^{\mathrm{e}}$ siècle) n'attachent que peu d'attention à la manière dont les différents acteurs peuplant chroniques et vitae instrumentalisent leur corps pour exprimer, moduler, cacher ou réprimer leurs affects. Il n'en demeure pas moins que l'articulation émotion/corps/geste est indubitablement dans le champ du lisible pour peu qu'on se prête au jeu de les dénicher. Il suffit de porter attention aux signifiants physiques tapis dans la trame de la narration pour saisir qu'ils renvoient souvent à des signifiés ou à des non-dits émotionnels. Sans qu'ils soient toujours explicitement indiqués par un substantif, des ressentis qui généralement se dérobent à l'appréhension première du lecteur viennent parfois s'inscrire de façon visible sur le corps. Toutefois, ces manifestations physiques de l'émotion appartiennent à deux registres différents.

1. P. KING, «Emotions in Medieval Thought», http://individual.utoronto.ca/pking/articles/ Emotions_in_Medieval_Thought.pdf (consulté le 01.03.2010).

2. D. L. SmaIL, «Emotions and Somatic Gestures in Medieval Narratives: The Case of Raoul de Cambrai », Zeitschrift für Literaturwissenschaft und Linguistik, 35 (2005), p. 34-48. 
Elles peuvent être spontanées et incontrôlées - relever du lapsus corporis - ou au contraire volontaires, publiques et souvent mises en scène, auquel cas elles relèvent de la performance, voire du performatif. Ce sont ces deux aspects de l'économie corporo-émotionnelle qui seront brièvement étudiés dans cet article. Dans un premier temps, nous analyserons l'émotion-lapsus corporis. Définissons d'abord le terme: lapsus corporis, tel que nous l'entendons, recouvre un champ dans lequel le corps, pris en otage par une émotion intense, se met à faire des confidences hors du contrôle de la volonté de celui qui en fait l'expérience. À l'instar d'une caisse de résonance amplifiant le vécu émotionnel, le corps devient l'unique médiateur de la mise en visibilité d'un affect que le sujet aurait préféré cacher aux autres ou dont il n'est pas totalement conscient lui-même. Rarement consentie par le sujet qui la ressent, la "publicisation" de l'émotionlapsus corporis n'en représente pas moins une forme de communication qui permet d'accéder à une connaissance approfondie des affects, et ce faisant elle vient éclairer dans sa plus grande concrétude le lien intime entre corps, émotion et intériorité. C'est cet aspect de l'économie corporo-émotionnelle, du simple geste somatique au sens où l'emploie Daniel Smail au phénomène de la conversion somatique, voire hystérique, qui sera abordé dans un premier temps.

Si certaines expressions permettent de pénétrer l'intériorité d'un personnage afin d'en restituer les émois, elles n'ont pas toutes cette vocation. Comme l'a souligné Jan-Dirk Müller ${ }^{3}$ dans son analyse du Nibelungenlied, l'émotion n'a pas systématiquement la dimension psychologique et privée que le sens commun lui prête en général. Les tenants de la communication symbolique, avec en tête de file Gerd Althoff, considèrent larmes et autres manifestations émotionnelles comme des éléments clés d'un système de communication bien réglé, et leur attribuent une place centrale dans les interactions politiques et dans le déroulement de rituels aussi différents que la reddition, la réconciliation ou la demande de faveur ${ }^{4}$. Dans cette perspective, les gestes émotionnels ne relèveraient plus du psychologique et du spontané mais d'une performance à visée stratégique, leur principale fonction étant de souligner, de moduler ou d'intensifier les actes rituels. Parallèlement à cette valeur de performance, au sens théâtral du terme, les gestes somatiques, tout comme les actes de langage définis par John Langshaw Austin ${ }^{5}$, ont également une dimension performative. Comme nous le verrons par la suite, un sourire ou des larmes peuvent représenter une émotion ritualisée, mais ils peuvent aussi signifier un défi, une provocation,

3. J.-D. MülLER, Rules for the Endgame. The World of the Nibelungenlied, Baltimore, 2007, p. $177-78$.

4. Voir note 27.

5. J. L. Austin, Quand dire, c'est faire, Paris, 1970. 
une menace, une promesse, et du même coup engendrer un changement dans la relation entre les interlocuteurs.

\section{Émotions-lapsus corporis : l'exemple des gestes somatiques}

Curieusement, alors que les manifestations physiologiques de certaines émotions telles que le rougissement, le pâlissement, le tremblement, sont les plus répandues et les moins culturellement marquées, leurs apparitions dans la littérature altimédiévale sont relativement rares. Il semble que les chroniqueurs et les hagiographes des premiers siècles médiévaux ne leur portaient qu'un intérêt modéré. Ce n'est donc qu'au prix d'une lecture tout en dentelle que l'on peut saisir, au détour d'une anecdote, ces gestes somatiques qui laissent transparaître des émois que l'on eût préféré cacher ou qui viennent trahir des sentiments intérieurs inavouables. Un premier exemple illustrera la place du somatique dans l'économie émotionnelle des hommes du haut Moyen Âge: le prélat Dubric vivait en compagnie d'un jeune diacre auquel il s'était fortement attaché. Voulant le garder auprès de lui, il pria saint Samson de lui accorder cette faveur. Le saint, qui avait la faculté de voir au-delà des apparences, scruta le jeune homme, l'embrassa et, après un bref silence, il répondit ainsi à Dubric :

Le filet, mon Père, recueille tous les poissons qu'il peut attraper au sein de la mer, mais ce n'est que lorsqu'il est tiré à terre que les pêcheurs séparent les bons des mauvais. En entendant cela, le prélat Dubric, car le diacre lui était très cher, gémit au fond de lui-même et cacha par son silence qu'il avait été blessé, mais sans pouvoir le dissimuler sur son visage (ita ut in suo vultu minime celare potuit). Saint Samson pour sa part, observa attentivement cette attitude, et comme s'il ne s'apercevait de rien, ordonna joyeusement de donner à ce même diacre pour apaiser plus facilement le prélat, le chrême ${ }^{6}$.

Ce bref extrait montre bien la fonction d'un corps parlant. Bien que Dubric désirât garder pour lui le sentiment qui le traversait, il ne put contenir son trouble, qui s'afficha malgré lui sur son visage. Nous sommes indubitablement en face d'un échec de la maîtrise émotionnelle aux prises avec un corps qui n'a cure du secret. Parmi les gestes somatiques, le rougissement est par excellence un dévoilement du quant-à-soi, processus par lequel l'invisible intériorité s'exhibe involontairement. Un deuxième exemple: soucieux de remplir les caisses du Trésor public, l'empereur Justinien s'adressa à Julienne, riche matrone, afin qu'elle lui prêtât une partie de sa fortune. Celle-ci, préférant vouer ses biens à Dieu plutôt qu'à l'Empire, lui répondit qu'elle ne possédait pas grand-chose mais que, s'il voulait bien attendre que les revenus de ses terres soient recueillis, elle

6. Vie ancienne de saint Samson de Dol, II, 7, éd. P. Flobert, Paris, 1997, p. 250-251. 
les présenterait à l'empereur afin qu'il puisse en disposer. L'empereur, floué par la ruse, s'en retourna chez lui, confiant. En attendant, la vieille dévote ordonna que tout l'or que contenaient ses coffres fût fondu et employé à la décoration de la voûte de l'église consacrée à Polyeucte. Une fois le travail terminé, elle fit appeler Justinien qui accourut tout joyeux à l'église. Et là, elle lui dit:

Ô très glorieux empereur, prends, je te prie, la voûte de cet édifice, et sache que tout mon pauvre bien a été employé dans cet ouvrage. Fais-en ce que tu voudras, je ne m'y oppose pas. Celui-ci levant les yeux, et admirant ce qu'il voyait, rougit (erubuit), et, pour cacher sa honte (ne pudor eius manifestaretur), loua l'ouvrage, remercia et se disposa à sortir ${ }^{7}$.

Tout empereur qu'il était, Justinien dut se rendre à l'évidence qu'il avait été dupé par une vielle femme mais, ne pouvant se soustraire à la marque visible de son embarras, il noya sa déconfiture dans un discours admiratif. Il s'agit donc bien d'un échec de la dissimulation d'une émotion. Parfois, le seul rougissement du visage suffit à véhiculer la honte. Un sous-diacre conçut le plan de voler des vases qu'il avait aperçus dans une crypte. Il entra un soir dans la basilique, prit quelques vases et chercha désespérément la sortie. Incapable de la retrouver, il erra toute la nuit. Le jour venu, toujours prisonnier, il se cacha pour éviter de ressentir la brûlure de la honte et cela trois nuits durant, jusqu'à ce que, torturé par la faim, il finisse par montrer son visage à la fenêtre. Rougissant (erubescendis), il confessa alors son méfait ${ }^{8}$. Compte tenu de la place de la honte dans l'économie émotionnelle du haut Moyen Âge, il est étonnant que ce geste somatique ne soit pas plus souvent relevé par les auteurs.

Toujours liée au répertoire de la honte et au plus près du corps, l'anecdote suivante, très suggestive, illustre le phénomène de la somatisation:

Lors de la fête de saint Jean, comme le peuple se rassemblait pour la célébration de la messe, une femme prit un sarcloir et alla au champ, afin de nettoyer la moisson en arrachant l'ivraie produite par la mauvaise semence; mais elle ne pouvait pas être fortifiée du secours divin, à cause du respect qu'il faut avoir pour le précurseur du Seigneur. Elle s'était mise à travailler, quand tout à coup ses mains furent saisies par un feu céleste, son visage parut comme lançant des flammes et se couvrit tout entier d'ampoules et de pustules. La malheureuse était

7. GRÉGOIRE DE Tours, Liber in gloria martyrum, 102, éd. B. KRuSCH, MGH SRM, I, 2, Hanovre, 1885, p. 106. La traduction est extraite de: H. L. Bordier, Les Livres de miracles et autres opuscules de Georges Florent Grégoire. Revus et collationnés sur de nouveaux manuscrits et traduits pour la Société de l'histoire de France, Paris, 1857-64, p. 277.

8. GRÉGOIRE De Tours, Liber in gloria martyrum, 37, p. 62. 
consumée, non moins par la douleur que lui causait la honte que de la douleur du corps, car elle révélait malgré elle ce qu'elle avait voulu faire secrètement ${ }^{9}$.

Ici, comme dans d'autres cas, la honte devient excroissance du corps qui montre à voir ce que l'on croyait bien caché. Mais avant de poursuivre plus avant, j'aimerais ouvrir une brève parenthèse.

Dans son article «Emotions and Somatic Gestures in Medieval Narratives », D. Smail suggère que «les protagonistes les plus enclins aux gestes somatiques sont exactement ceux qui sont les plus nobles, les plus dignes d'admiration ${ }^{10} »$. Contrairement aux narrateurs plus tardifs, les auteurs du haut Moyen Âge n'attribuent pas aux signes somatiques le caractère noble que leur confèrent les chanteurs de gestes. Pour les auteurs altimédiévaux, et tout particulièrement pour Grégoire de Tours, l'extériorisation involontaire des émotions est perçue comme une défaillance du contrôle sur soi, ou pis, comme un défaut d'intégrité. La dissonance entre l'émotion et le corps est considérée comme le comble de l'immoralité et de l'inélégance. Qu'elles soient métaphoriques ou concrètes, les manifestations physiques de l'émotion contribuent à la construction de l'image d'une aristocratie indigne, confinée dans un corps en perpétuel émoi que la raison et la volonté ne sauraient policer. Des gestes somatiques à la somatisation, processus par lequel apparaissent des symptômes physiques résultant de conflits émotionnels, le pas est aisément franchi.

\section{Émotions-lapsus corporis et les théâtres du corps}

Tout en empruntant ce beau titre à Joyce McDougall ${ }^{11}$, j'hésite à accepter sa séduisante invitation à explorer certains phénomènes psychosomatiques du haut Moyen Âge à la lumière de la psychanalyse. Michel Rouche, quant à lui, est persuadé de la validité de l'approche:

Bien des névroses expliquent certaines paralysies, comme les mains serrées sur la paume au point que les ongles finissent par la traverser et nombre de déficiences sensorielles. Mais il s'y ajoutait des névroses hystériques avec dédoublement de la personnalité, des états maniaques, accompagnés de logorrhée, souvent d'origine alcoolique ${ }^{12}$.

9. GrÉGOIRe De Tours, Libri de virtutibus, II, 57, éd. B. Krusch, MGH SRM, I, 2, Hanovre, 1885, p. 178. Traduction: Les Livres de miracles..., p. 185.

10. D. SmaIL, «Emotions and Somatic Gestures...», p. 43.

11. J. McDougall, Théâtres du corps : le psychosoma en analyse, Paris, 1989.

12. M. Rouche, «Le haut Moyen Âge occidental», dans P. ArIÈs et G. Duby éd., Histoire de la vie privée, t. 1, Paris, 1985, p. 399-530 (ici p. 461). 
Mon hésitation ne relève pas tant d'une méfiance à l'égard de la métapsychologie que de la difficulté à appliquer ce type de grille de lecture à des textes historiques sans tomber dans les travers d'un psychologisme excessif. Dans la littérature du haut Moyen Âge, derrière le corps ému signalant discrètement une intériorité qui rechigne à se manifester s'en dissimule parfois un autre, le corps théâtral, incontrôlé et inconvenant, que certains auteurs se prêtent volontiers à décrire avec force détails tant ils semblent fascinés par ses déchaînements. Ce corps qui ne tient pas en place, qui s'emballe dans une gestuelle proche de l'hystérie, avec ses gestes convulsifs et ses interludes de mutisme, de cécité ou de paralysie, ce corps parfois malade et souffrant, offre un fascinant spectacle où émotionnalité et corporéité fusionnent au plus près. Toutefois, alors que dans l'émotion-lapsus corporis le rapport émotion/corps est manifeste, les épisodes de conversion somatique ne font aucune référence à un vécu émotionnel clairement énoncé - les traduire en langage émotionnel relèverait de la gageure. Cependant, sans se référer à une émotion particulière, on pourrait fort bien parler en termes d'intériorité. Au même titre que les gestes somatiques divulguent telle ou telle émotion, les conversions somatiques ont pour fonction de rendre claire une intériorité que l'on veut taire. Partie intégrante de la rhétorique hagiographique, la projection de l'intériorité à l'extérieur apparaît souvent dans les scènes de parjure où le fauteur, désirant dissimuler son méfait ou son mensonge, est dénoncé par son propre corps. En voici quelques exemples. Une jeune femme se rend un jour au marché pour y faire des emplettes, un objet attire son attention. Le marchand le lui tend afin qu'elle l'examine de plus près; aussitôt, elle le passe à un complice et nie l'avoir reçu en mains propres. Pressée par le marchand de prêter serment pour prouver son innocence, elle relève le défi et se dirige, suivie de son accusateur et d'une foule de badauds, vers le tombeau de saint Eugène:

Comme elle avait les mains levées pour jurer, tout à coup elle devint raide comme si ses membres eussent été perclus, la plante de ses pieds resta fixée au pavé, sa voix s'arrêta dans son gosier, et elle resta la bouche béante sans pouvoir proférer un $\operatorname{mot}^{13}$.

Elle resta fort longtemps dans cette position douloureuse jusqu'à ce que le martyre la fît parler. Un homme commet un larcin dans une église. Un an après, jour pour jour, sa tête enfle et ses yeux se gonflent tant qu'ils semblent prêts à sortir de leurs orbites. Ce mal lui revient chaque année au jour de son vol ${ }^{14}$. Les cas de femmes dont les membres se dessèchent car elles ont osé pétrir et cuire du pain le dimanche relèvent presque du topos. Un jour, une femme perdit l'usage de ses membres pour ce même motif:

13. GRÉGOIRE DE TouRs, Liber in gloria martyrum, 57, p. 78.

14. Ibid., 58, p. 78. 
Comme elle mettait un pain au four un samedi après le coucher du soleil, son bras fut saisi d'une douleur. Elle jeta dans le four un second et un troisième pain; alors sa main commença à serrer involontairement le bois qu'elle tenait: la femme, comprenant qu'elle était condamnée par un jugement de la puissance divine, rejeta promptement la pelle qu'elle tenait. Néanmoins, elle ne put échapper à sa punition ${ }^{15}$.

En se transformant en loci de vérité, ce bras douloureux, ces membres desséchés, cette tête et ces yeux enflés font prendre conscience aux fauteurs que leurs actes sont illicites et moralement répréhensibles. Le corps aurait presque une fonction disciplinaire, garde-fou à l'égard des dérives du comportement et des sentiments secrets et transgressifs. Ces signes somatiques semblent bien relever de la conversion hystérique.

Pour les hagiographes, l'enveloppe corporelle est à la fois un écran à l'authentique et une entité autonome qui a la capacité de rendre visibles les secrets de la vie intérieure. Souvent sous l'impulsion d'une intervention supranaturelle, revêtant une forme diabolique, le corps se met à parler. Comme le dit Barbara Rosenwein, le diable éprouve aussi des sentiments, et même s'il en est dépourvu, il a le don de les inspirer à ses victimes ${ }^{16}$. Dans le discours des auteurs, le diable est à la convergence du corps et de l'émotion. Connu de la littérature médiévale comme l'instigateur des phénomènes de possession, le diable et ses stratégies démoniaques ont été largement étudiés par les historiens et les psychologues, et bien qu'il y ait une certaine banalité à rapprocher les turpitudes du diable du phénomène de la conversion hystérique, je ne peux pourtant renoncer à poursuivre l'analogie. Un seul exemple suffira. Piqué de jalousie à l'égard de saint Samson, un moine tenta en vain de l'empoisonner. La vengeance divine ne tarda pas à s'abattre sur le moine envieux :

Effectivement, quand vint le dimanche suivant, après avoir reçu la communion à l'autel de la main de saint Samson, il fut saisi par le démon ( $a$ demonio... arreptus est) à l'instant même où l'hostie pénétrait dans sa bouche, si bien que, pâlissant et tremblant (pallens tremensque), lâchant une série de pétarades et se déchirant lui-même, il tomba sur le sol d'une façon indécente (indecenter) et, se dénudant lui-même sans pudeur (impudenter) de ses vêtements, il se mit à se mordre les deux lèvres avec les dents (dentibus mordere), à bafouer les autres moines sans aucune considération (sine verecundia) de respect et à provoquer leurs cœurs à la colère ${ }^{17}$.

15. GrÉGoIre de Tours, Libri IV de virtutibus S. Martini, III, 57, éd. B. Krusch, MGH SRM, I, 1, Hanovre, 1951, p. 235, voir p. 261 et 263.

16. B. H. Rosenwein, «Even the Devil (Sometimes) Has Feelings: Emotional Communities in the Early Middle Ages», The Haskins Society Journal : Studies in Medieval History, 14 (2003), p. 1-14.

17. Vie ancienne de saint Samson de Dol, I, 18, p. 175-176. 
Image de la transmutation de l'intériorité en gestes indécents et en actes insensés, le diable, «métaphore du négatif ${ }^{18}$ » psychologique et corporel, participe directement de l'économie émotionnelle dans ce qu'elle a de plus corporel et de plus thêâtral. Selon l'analyse de Luisa de Urtubey, le diable remplit deux fonctions essentielles dans la théorie freudienne. Il représente l'inconscient et les pulsions refoulées mais il est aussi «métaphore de la contre-volonté (responsable, dit Freud, du caractère diabolique exhibé si souvent par l'hystérie, quand les patients ne peuvent faire ce qu'ils souhaitent tandis qu'ils se sentent obligés à agir à l'opposé de leur volonté consciente, contrainte qui a les mêmes caractères de la possession diabolique) ${ }^{19}{ }^{1}$. Par l'effet d'un dénudement indécent du corps, celui qui désirait préserver son intériorité s'expose tout entier et sans dissimulation dans son appareil le plus modeste et par là donne à voir ce que la conscience était incapable d'exprimer par la parole, à savoir la faute, le péché, la transgression. La vérité nauséabonde sort dans un grand bruit de pétarades. Ne pouvant mentir, le corps devient preuve d'authenticité. À la convergence de l'expérience émotionnelle vécue et de sa publication involontaire et incontrôlée se profile donc un «corps éloquent ${ }^{20}$ », unique vecteur de l'intériorité qui se projette entièrement au-dehors de soi, sans travestissement ni dissimulation ou, plus exactement, malgré le travestissement et la dissimulation. Dans ce cas de figure, le diable, évoqué comme une métaphore et non comme une entité concrète et autonome, se fait le complice de Dieu pour mieux punir le fauteur.

Mais il est temps de passer au second aspect de l'économie corporoémotionnelle. Comme nous le mentionnions dans l'introduction, les gestes somatiques ne possèdent pas systématiquement la propriété de lier intérieur et extérieur. Ils peuvent également relever de la mise en scène.

\section{Les larmes de Frédégonde: entre rituel et performativité}

Dans une excellente étude publiée en 2003, Kathryn Starkey s'attachait à décoder le sourire qu'adresse à ses adversaires la reine Brunehilde, l'une des figures les plus saillantes du Nibelungenlied. Replacé dans son contexte, il s'avère que ce simple rictus est un formidable révélateur d'informations sur la culture émotionnelle que véhicule l'ensemble de la chanson de geste et sur la fonction des gestes somatiques à la fois dans la trame narrative et dans les

18. L. De Urtubey, «Les ruses et les pièges du Diable», dans J. Guillemin éd., Pouvoirs $d u$ négatif dans la psychanalyse et la culture, Paris, 1988, p. 74-80 (ici p. 77).

19. Ibid., p. 76.

20. Expression empruntée à L. Desjardins, Le Corps parlant. Savoirs et représentations des passions au XVII siècle, Québec-Paris, 2001, p. 103. 
rapports sociaux ${ }^{21}$. S'appuyant sur le point de vue de Jan-Dirk Müller selon lequel, dans le Nibelungenlied, l'émotion ne viendrait pas systématiquement exprimer un affect mais opérerait un changement, voire une rupture, dans une situation, Kathryn Starkey suggère que les gestes émotionnels obéiraient aux mêmes règles. Ces derniers auraient non seulement une fonction communicative - en ce sens elle corrobore la thèse de Gerd Althoff -, mais ils posséderaient la propriété «d'affecter un état des choses socialement reconnu, changeant le statut de quelqu'un ou de quelque chose ${ }^{22} \gg$. La notion de performativité estelle pertinente dans le contexte général de la littérature mérovingienne et plus spécifiquement dans le cas des larmes de Frédégonde? Sont-elles la reproduction d'un simple geste rituel stéréotypé ou représentent-elles une stratégie infiniment plus complexe dans la fabrique des relations de pouvoir? Ce n'est qu'en étudiant, comme le suggère Gary Ebersole, les performances individuelles plutôt que des scénarios standardisés que l'on pourra restituer les motivations personnelles des acteurs et rendre à ces pratiques impersonnelles leur dimension transgressive, productive et performative ${ }^{23}$. Avant de tenter de répondre à ces questions, reportons-nous au récit que fait Grégoire de Tours des circonstances qui ont engendré ces larmes.

Ayant fait courir le bruit que Frédégonde entretenait une relation adultère avec l'évêque Bertrand, Leudaste, comte de Tours, s'attire l'hostilité non seulement de l'épiscopat, qui l'excommunie pour avoir calomnié un de ses membres, mais encore du roi et de la reine: «Incriminer ma femme, dit le roi, c'est me déshonorer!» Par ordre royal, le détracteur est battu, destitué de son honor et emprisonné. Mais l'incarcération est de courte durée. En butte à différentes pressions de l'entourage royal, Frédégonde, bien à contre gré, se voit dans l'obligation de libérer son calomniateur. La teneur du message qu'elle envoie à Grégoire au sujet de Leudaste indique bien que pour elle l'incident est loin d'être clos, et laisse présager une vengeance imminente. Leudaste, quant à lui, n'est conscient de rien. Non content d'avoir été si rapidement libéré, il se met en tête de récupérer son ancien statut. Pour cela, il lui faut recouvrer les bonnes grâces du couple royal. Au terme de multiples démarches auprès des membres de la trustis royale, il se voit finalement accorder une audience. Prosterné en signe d'humilité, le comte déchu obtient du roi le pardon tant désiré. Mais cette mansuétude n'est pas partagée par la principale intéressée qui, elle, ne décolère

21. K. Starkey, «Brunhild's Smile. Emotion and the Politics of Gender in the Nibelungenlied», dans C. S. JAEGER et I. KASTEN éd., Codierungen von Emotionen in der Kultur und Literatur des Mittelalters und der frühen Neuzeit: Paradigmen und Perspektiven, Berlin, 2003, p. 159-173, p. 173.

22. K. StaRkey, «Brunhild's Smile...», p. 163. (Ma traduction.)

23. G. Ebersole, «The Function of Ritual Weeping Revisited: Affective Expression and Moral Discourse», History of Religions, 39 (2000), p. 211-246 (ici p. 240-241). 
pas. Connaissant l'état d'esprit de son épouse, Chilpéric conseille d'ailleurs à Leudaste d'éviter la reine. Il la consulterait d'abord, et ils conviendraient ensuite de la manière dont Leudaste pourrait l'aborder sans s'attirer ses foudres. Enhardi par son entrevue avec le roi, il fait fi du conseil. Un dimanche, au moment solennel de la messe, en plein milieu d'une église probablement bondée, il se jette aux pieds de la reine pour demander pardon:

Mais celle-ci, grinçant des dents (frendens) et maudissant sa vue (execrans aspectus eum), le repoussa loin d'elle et fondant en larmes (fusisque lacrimis) s'écria: «Puisqu'il ne me reste plus de fils pour instruire l'affaire dont on m'accuse, c'est à toi Jésus mon seigneur, que j'en confie l'instruction.» Puis s'étant prosternée aux pieds du roi (postrataque pedibus regis), elle ajouta: «Malheur à moi qui vois mon ennemi et ne puis point l'emporter sur lui.» ${ }^{24}$

Une fois Leudaste chassé, on continue la messe. Satisfait de son initiative, ne soupçonnant pas ce qui va lui arriver, il s'en va au marché, le cœur léger, vantant à qui veut bien l'entendre le recouvrement prochain de sa fortune. Mais la vengeance de la reine ne se fait pas attendre. Elle envoie ses sicaires pour se saisir de Leudaste, qui est blessé au cours de l'opération. Sur l'ordre de la reine, il est jeté à terre et tandis qu'il est couché sur le dos, maintenu par une immense barre de fer, on lui frappe la tête avec une autre jusqu'à ce que mort s'ensuive.

L'extrait est considérablement riche et complexe car il articule dans un ensemble apparemment cohérent des paroles, des gestes et des expressions émotionnelles liés à différentes pratiques rituelles. Cernons d'abord les gestes et les expressions du visage pour ensuite les replacer dans leur cadre rituel. La scène débute avec le grincement des dents, qui marque une forme de courroux. Alors que d'autres locutions associées à la colère comme felle commotus, felle fervens, felle succensus suggèrent un sentiment plus intériorisé rendu par l'image du bouillonnement de la bile, "grincer des dents » possède une dimension visuelle indispensable à la performance. Une analyse des différents contextes dans lesquels apparait le mot frendens ${ }^{25}$ montre qu'il est souvent accompagné de proférations hostiles. Tout en grinçant des dents, on se répand en menaces, on vocifère, on maudit, on vomit des blasphèmes. Paul Diacre utilise dans le même souffle les termes frendens et comminans ${ }^{26}$, ce dernier dans un sens de

24. GréGoire de Tours, Libri historiarum X, VI, 32, éd. B. Krusch et W. Levison, MGH SRM, I, 1, Hanovre, 1951, p. 303. Traduction française: GRÉGOIRE DE Tours, Histoire des Francs, éd. R. Latouche, Paris, 1995.

25. GréGoire de Tours, Vitae Patrum, 9, B. Krusch éd., MGH SRM, I, 2, Hanovre, 1885, p. 253 ; Libri Historiarum X, III, 31, p. 126; IV, 20, p. 153; V, 43, p. 252; VI, 32, p. 303; Liber in gloria confessorum, 66, p. 337.

26. Paul Diacre, Historia Langobardorum, V, 39, G. Waitz éd., MGH SRL, vol. 48, Hanovre, 1878, p. 204. 
démonstration menaçante. Le grincement des dents vient donc traduire une colère déclarative qui se doit d'être arborée puisque son but est principalement d'intimider ou de faire peur. Frédégonde, ici, a deux bonnes raisons de montrer les dents. Leudaste l'a doublement agressée, d'abord en la calomniant, ensuite en transgressant l'étiquette formelle de la supplique qui exige de «demander informellement si les seigneurs étaient éventuellement disposés à entendre tel ou tel vœu ${ }^{27} \gg$. C'est donc sous les auspices de la menace et de l'hostilité que débute l'incident. Immédiatement après ses grincements de dents qui viennent avertir Leudaste et l'assemblée tout entière qu'il vaut mieux ne pas se frotter à elle, Frédégonde change subitement de registre: elle s'effondre en larmes, se jette aux pieds de Chilpéric et se lamente.

Le geste de prosternation rappelle le rituel de la supplique, qui a déjà fait l'objet de nombreuses recherches dont la plus connue est celle de G. Koziol ${ }^{28}$. Rappelons simplement que ce rituel est largement présent dans les interactions politiques et sociales du Moyen Âge. Au cours de la cérémonie, «le suppliant s'astreignait à une posture corporelle manifestant l'humilité, s'agenouillait voire s'allongeait sur le sol, et il n'était pas rare qu'il exprimât sa supplique uniquement par une prosternation muette ${ }^{29} »$. Parfois, des «personnes de rang élevé, et même des rois, utilisaient également ce moyen pour conférer une importance particulière à leurs réclamations ${ }^{30} »$. À la prosternation s'ajoutent les larmes. Dans la culture médiévale, les gens versaient des larmes pour toutes sortes de raisons. Certaines étaient de nature religieuse, comme le don des larmes étudié par Piroska $\mathrm{Nagy}^{31}$. D'autres encore étaient spontanées comme les larmes d'impuissance de Gundovald et de Mérovée, ou elles pouvaient, au contraire, faire l'objet d'une mise en scène rituelle. Selon Gary Ebersole, les larmes rituelles «sont un acte culturellement chorégraphié, une expression stylisée des émotions ${ }^{32} »$, qui ne sauraient se passer d'auditoire. Les larmes de Frédégonde, à l'évidence, n'ont rien de spontané. Elles sont fréquemment utilisées par des personnes sans défense, et représentent les «seules armes», pour reprendre l'expression de Scott, «qui pouvaient parfois obliger un supérieur social à jouer son rôle dans

27. G. Althoff, «De l'importance de la communication symbolique pour la compréhension du Moyen Âge», Trivium, 2 (2008), http://trivium.revues.org/index992.html. Consulté le 28 septembre 2010.

28. G. KozIoL, Begging Pardon and Favor. Ritual and Political Order in Early Medieval France, Ithaca/Londres, 1992.

29. G. Althoff, «De l'importance de la communication... », p. 13.

30. Ibid., p. 13.

31. P. NAGY, Le Don des larmes au Moyen Âge, Paris, 2000.

32. G. Ebersole, «The Poetics and Politics of Ritualized Weeping in Early and Medieval Japan», dans K. C. Patton et J. S. Hawley éd., Holy Tears. Weeping in the Religious Imagination, Princeton, 2005, p. 26. 
une relation socialement et moralement sanctionnée ${ }^{33} \gg$. Mais Frédégonde ne se contente pas de se prosterner et de pleurer. Elle ajoute des paroles aux gestes, et son discours est sans ambiguïté. Derrière son désespoir se dessine un véhément appel à la vengeance. Ses reproches évoquent à plus d'un égard le rituel mis en œuvre par la goading woman $^{34}$, la fameuse figure de proue de la littérature islandaise. Dans une atmosphère où honneur et rétorsion sont étroitement liés, le rôle de la femme incitatrice est de rappeler à sa parenté mâle, en usant parfois d'invectives, le devoir qui lui incombe en matière de vengeance ${ }^{35}$. Ce type de cérémoniel, également pratiqué par les Mérovingiennes, transparaît clairement dans les incitations à la vengeance que prononce Clotilde à l'attention de ses fils, les priant instamment de laver la mort de ses parents dans le sang ${ }^{36}$. En adoptant l'attitude de la femme incitatrice, Frédégonde manifeste son désir de vengeance et fait aveu d'impuissance.

Deux remarques s'imposent ici. Toutes les expressions émotionnelles auxquelles a recours Frédégonde relèvent de la performance. Elle alterne la colère et les larmes, puise simultanément dans les registres de la supplique et de l'incitation à la vengeance, preuve s'il en est besoin que sa spontanéité est pour le moins aléatoire. En outre, tous ces gestes sont manifestement destinés à être vus et entendus par l'ensemble de l'assistance autant que par le roi.

La seconde remarque a trait à l'économie de la performance de la reine. La surenchère des gestes, la dramatisation toujours plus intense et le chevauchement des rituels sont un indice que les enjeux dépassent la seule volonté d'inciter à la vindicte ou de susciter la compassion de Chilpéric pour qu'il passe à l'action dans le sens d'une rétorsion. Pour bien comprendre ce qui est en jeu, il faut saisir la manière dont Frédégonde use des gestes symboliques que la culture met à sa disposition et recompose la chorégraphie rituelle afin de véhiculer son propre message.

Prenons par exemple le rituel de l'incitation à la vengeance. L'usage qu'en fait Frédégonde permet de mettre en relief un certain décalage par rapport au modèle «traditionnel». Les cérémoniels de demande de vengeance s'échafaudent généralement à partir de trois éléments: la femme qui crie vengeance, la personne à qui elle adresse sa requête et le corps de la victime, ou un symbole le représentant, comme pièce à conviction de l'offense ${ }^{37}$. Dans le cas présent,

33. J. Sсотт, Weapons of the Weak Everyday Forms of Peasant Resistance, New Haven et Londres, 1985.

34. La figure de la goading woman, la femme incitatrice, a été étudiée par J. M. JocHENs, « The Medieval Icelandic Heroine : Fact or Fiction? », Viator, 17 (1986), p. 35-50.

35. I. Miller, «Choosing the Avenger : Some Aspects of the Bloodfeud in Medieval Iceland and England», Law and History Review, 1 (1983), 159-204.

36. GréGoIre de Tours, Libri Historiarum X, III, 6, p. 103.

37. I. Miller, «Choosing the Avenger... », p. 181. 
il n'est pas question de cadavre puisqu'aucun assassinat à proprement parler n'a eu lieu. Il s'agirait plutôt de l'assassinat de sa réputation et du cadavre de son honneur. La subversion de l'usage des gestes somatiques ne s'arrête pas là.

Comme nous l'avons déjà remarqué précédemment, le rituel de la supplique - mais on peut estimer que ce principe est essentiel dans tout type de rituel - exige l'accord préalable de celui qui est censé octroyer la faveur. Or, en se jetant aux pieds de Chilpéric, Frédégonde reproduit l'insulte que lui a faite Leudaste. Elle bafoue l'honneur de Chilpéric tout comme Leudaste l'a outragée. En escamotant délibérément le protocole du rituel, la reine signifie une rupture de l'ordre normal des choses. Une autre particularité a trait aux larmes ou plutôt à leur destinataire. Les larmes rituelles non religieuses sont toujours adressées à un supérieur hiérarchique et ont pour principale fonction de marquer des relations hiérarchiques entre les individus ${ }^{38}$. On pourrait donc penser qu'en offrant ses larmes à Chilpéric, Frédégonde déclare publiquement son infériorité et sa dépendance vis-à-vis de son royal mari. Or elle n'en fait rien. Ce n'est ni au roi ni au mari qu'elle s'adresse en pleurant pour instruire son affaire, mais directement à Dieu, auquel elle offre le témoignage de son humilité. En refusant d'adresser ses larmes à son compagnon, elle l'exclut non seulement du rituel en tant qu'agent actif mais refuse de lui reconnaître l'autorité et la supériorité qui lui reviennent en tant qu'homme, en tant que mari et en tant que roi. Chilpéric est donc sciemment ignoré par Frédégonde pendant toute la durée de l'incident. Ce n'est que dans la dernière phase du rituel qu'elle se tourne finalement vers lui. En se jetant à ses pieds, elle profère une phrase dont la teneur est ambiguë à plus d'un égard: «Malheur à moi qui vois mon ennemi et ne puis point l'emporter sur lui.» À qui s'adresse-t-elle véritablement? à Chilpéric? à Dieu ? à l'auditoire? à Leudaste ? Et surtout, quel en est le ton ? Exprime-t-elle un réel désespoir, une colère mêlée d'indignation ou un reproche à Chilpéric?

Pourquoi la reine consent-elle à s'humilier alors qu'en fait, dès le début de l'affaire, sa vengeance est en suspens telle une épée de Damoclès au-dessus de la tête de Leudaste? Grégoire en est très conscient puisque, craignant que la reine ne le fasse assassiner, il lui suggère de se tenir sur ses gardes. La vindicte de la reine est donc en gestation depuis l'instant où Leudaste l'a faussement accusée. Elle attend simplement le moment opportun, et Leudaste lui offre cette occasion au moment de la messe dominicale. La scène de l'église, qui présente une Frédégonde blessée, meurtrie et déshonorée, prenant Dieu à partie de son infortune, relève d'un bout à l'autre de la mise en scène. Frédégonde est une maîtresse femme à qui le qualificatif de victime ne sied pas. Son attitude ne révèle en rien une faiblesse typiquement féminine. Au fond, elle n'avait nul besoin

38. G. Ebersole, «The Poetics and Politics of Ritualized...», p. 26. 
de toute cette mise en scène pour exécuter son projet d'assassinat, qui semble planifié dès le début de l'incident. Connaissant sa superbe, son sens du pouvoir et son autonomie en matière d'assassinats politiques, elle est en position de se passer de l'assistance de Chilpéric. Le dénouement de l'histoire en est la preuve formelle puisque c'est sur son ordre que ses sicaires vont appréhender Leudaste.

Deux interprétations sont possibles. La première, la moins crédible, est que Frédégonde a besoin de l'aval du roi pour que le meurtre qu'elle est sur le point de faire perpétrer soit accepté comme la juste et légitime rétorsion de son offense - après tout, le roi ayant accordé son pardon à Leudaste, l'initiative non concertée de sa femme aurait pu être considérée comme un acte de désobéissance. Ce serait donc là une manifestation de sa soumission vis-à-vis de son royal mari. Mais eu égard aux agissements passés de Frédégonde, cette hypothèse semble peu probable. Le second scénario possible est que toute cette mise en scène soit destinée au contraire à reprocher publiquement à son mari son indulgence vis-àvis de Leudaste, et surtout à lui rappeler le sens des priorités. Dans le déroulement normal de la cérémonie, les larmes rituelles auraient dû être adressées à son mari, qui en l'absence de fils aurait dû se charger de la venger. Mais elle choisit de les offrir à Dieu. Quant au caractère humiliant de la prosternation, il est partiellement annulé par la phrase qui l'accompagne puisque cette dernière remet en cause la capacité de son mari à défendre son honneur. En effet, quel est le malheur de Frédégonde sinon celui de voir son ennemi jouissant de sa liberté alors qu'elle souffre encore les affres de l'humiliation du fait du manquement de son mari?

Sa mise en scène, à mi-chemin entre le rituel et la stratégie, aurait donc une valeur performative, dont l'objectif serait de faire publiquement honte à Chilpéric et de lui rappeler que rien ne se décide sans elle. Dans son one woman show, Frédégonde joue simultanément le rôle de la victime à venger, celui de l'incitatrice à la vengeance et, finalement, celui de l'exécutrice de la vengeance. C'est là une première réappropriation, ou une première instrumentalisation, d'un rituel existant. Ce qui semblait être à première vue un rituel humiliant s'avère en fin de compte une démonstration de pouvoir. Ainsi, au même titre que pour le sourire décrit par Kathryn Starkey, on pourrait voir dans les larmes publiques de Frédégonde une dimension performative qui dépasse de loin la simple communication symbolique. Elles annoncent l'exécution prochaine de sa vindicte et le rétablissement de l'ordre et de la justice dans l'aula regis, tout en dénonçant la pusillanimité de son royal mari.

À la croisée entre le psychologique et le social, les gestes somatiques sont multifonctionnels. Qu'ils soient spontanés ou rituels, performants ou performatifs, les gestes somatiques participent d'un système de communication extrêmement complexe et nuancé. Les quelques exemples et réflexions présentés dans cette étude préliminaire suggèrent qu'on ne saurait les ignorer sous peine de se priver d'une formidable source d'information sur la manière dont les hommes 
et les femmes du haut Moyen Âge utilisaient leur corps pour exprimer, moduler, cacher ou réprimer leurs affects.

Nira PANCer - University of Haifa, Departement of History, Mount Carmel, Haifa 31905, Israël

Entre lapsus corporis et performance: fonctions des gestes somatiques dans l'expression des émotions dans la littérature altimédiévale

La littérature des premiers siècles médiévaux (VI-VIII ${ }^{\mathrm{e}}$ siècle) attache peu d'attention aux émotions, et a fortiori aux gestes somatiques qui les traduisent. Cependant, malgré leur relative rareté dans les textes, ces derniers n'en jouent pas moins un rôle capital dans la fabrique des relations sociales et dans les négociations de pouvoir. C'est la triple fonction de ces gestes proprement émotionnels qui sera examinée ici. Certains, tels que le rougissement, sont des lapsus corporis qui, échappant à toute maîtrise, viennent révéler une intériorité inavouable. D'autres encore, ritualisés et normés, relèvent de la «performance émotionnelle» dans le sens goffmanien du terme. En dernier lieu, certains sourires ou larmes possèdent une dimension performative. Ils ont alors la capacité d'affecter les liens sociaux et d'instaurer de nouveaux rapports de force entre les interlocuteurs.

Émotions - gestes somatiques - performance - performatif - Mérovingiens.

Between lapsus corporis and performance: the functions of somatic gestures in the expression of emotions in the early Middle Ages literature

Early medieval literature shows little interest in emotions, let alone in the somatic signs translating them. Nevertheless, despite their relative scarcity in the texts, these somatic gestures do play a major part in the making of social relationships and in power negotiations. This article will examine their threefold function. Some of them, escaping control, such as blushing, are lapsus corporis, revealing a shameful inwardness. Others, ritualized and normative, are similar to emotional performance in the Goffmanian tradition. At last, certain smiles or tears possess a performative effect changing social relationships and establishing a new balance of power between speakers.

Emotions - somatic gestures - performance - performative - Merovingians. 
